\title{
Role of the autophagy-related marker LC3 expression in hepatocellular carcinoma: a meta-analysis
}

\author{
Yu-Chen Meng ${ }^{1} \cdot$ Xiao-Li Lou ${ }^{1} \cdot$ Li-Yuan Yang $^{1} \cdot$ Dong Li $^{2} \cdot$ Yan-Qiang Hou ${ }^{1}$ (i)
}

Received: 30 December 2019 / Accepted: 2 March 2020 / Published online: 10 March 2020

(c) The Author(s) 2020

\begin{abstract}
Background Microtubule-associated protein 1 light chain 3 (LC3), an autophagic gene, has been reported as a vital marker for many diseases and cancers. However, the role of LC3 in hepatocellular carcinoma (HCC) was not still investigated. Therefore, we conducted a meta-analysis to examine the association of LC3 with its clinicopathological and prognostic in HCC. Methods We consulted the PubMed, Cochrane Library, Web of Science, EMBASE, China National Knowledge Infrastructure and Wan Fang databases for published studies on LC3 in HCC. Newcastle-Ottawa scale was used to screen the quality of the literature. The statistical analysis was calculated by STATA 14.2.

Results Of the 1329 titles identified, 10 articles involving 949 patients in HCC were included in this meta-analysis. The results of our study show that increased LC3 expression is related to size of tumor, but not to gender, age, number of tumor, liver cirrhosis, HBsAg, TNM stage, alpha fetoprotein, vascular invasion and histological grade. Positive LC3 expression was associated with overall survival by pooled hazard ratio.

Conclusions This meta-analysis indicated that positive LC3 expression was related to size of tumor, and could predict prognosis in human hepatocellular carcinoma.
\end{abstract}

Keywords LC3 $\cdot$ Hepatocellular carcinoma $\cdot$ Meta-analysis $\cdot$ Prognosis

\section{Introduction}

Hepatocellular carcinoma (HCC) is the third leading cause of cancer-related death and the fifth most common incidence rate (Yao et al. 2019), and it has a poor prognosis (Liang et al. 2018). About 800,000 of new cases of HCC in each year were examined worldwide, People's Republic of China count more than 50\% of these cases (Gingold et al. 2018). Currently, HCC was treated with surgery, chemotherapy, radiotherapy and sorafenib-targeted drug therapy (Hartke et al. 2017; Boyvat 2017). Although the treatment measures

Dong Li

lidong@tongji.edu.cn

$\triangle$ Yan-Qiang Hou houyanqiang@aliyun.com

1 Department of Central Laboratory, Songjiang Hospital Affiliated First People's Hospital, Shanghai Jiao Tong University, 746 Zhongshan Road, Shanghai 201600, China

2 Department of Laboratory Medicine, Tongji Hospital of Tongji University School of Medicine, 389 Xincun Road, Shanghai 200065, China have made a great progress recently, the clinical cure rate is still poor, especially number of patients miss the surgery because of the lack of early detection and treatment (Grandhi et al. 2016). Therefore, developing effective early predictive marker is necessary to HCC.

Autophagy is an intracellular catabolic process which contributes to homeostasis, differentiation, recycling of damaged organelles or long-lived proteins (Lee and Jang 2015; Han et al. 2014). Increasing evidences have demonstrated that autophagy is associated with tumor development and progression (Lv et al. 2019). Several proteins are involved in the human autophagic pathway, the microtubule-associated protein 1 light chain 3 (LC3) and Beclin-1 play an important role which have been reported to associate with the physiology and pathogenesis of human liver disease (Chih et al. 2017).

LC3, the mammalian homolog of the yeast Atg8p, is considered as the crucial component of autophagosomes (Maruyama et al. 2014). It includes three isoforms of LC3A, LC3B, LC3C, of which LC3B is associated with autophagy levels (Wu et al. 2014a). LC3 has been reported in several tumors (including ovarian cancer (Shen et al. 
2008), brain cancer (Ghavami et al. 2014), colorectal cancer (Wu et al. 2015), prostate cancer (Zhang et al. 2017), breast cancer (Jiang et al. 2017), melanoma (Segala et al. 2017). A meta-analysis of LC3 and ovarian cancer has shown that LC3 expression is associated with FIGO stage (Zhao et al. 2017). LC3 expression has also been found to show controversial results in the clinicopathological features in HCC patients (Lee et al. 2013; Bao et al. 2014; Ding et al. 2008). Wu et al. (2015) have indicated that LC3 expression is significantly associated with vascular invasion; high LC3 expression had indicated a poor overall survival (OS). However, Wu et al. (2014b) have found that LC3 expression is significantly correlated with male gender, large tumor size, tumor stage and worse relapsefree and OS. Chih et al. (2017) have demonstrated that the absence of LC3 expression is strongly predictive of immediate mortality for HCC. Hence, we conducted a meta-analysis to explore the relationship between LC3 expression and its clinicopathological characteristics and prognostic value in HCC.

\section{Methods}

\section{Search strategy}

A literature search of the PubMed, Web of Science, Cochrane Library, EMBASE, Chinese China National Knowledge Infrastructure (CNKI) and Chinese WanFang was performed to English and Chinese articles published before September 2019 using combinations of the following search terms: "LC3" or "microtubule-associated protein 1 light chain 3" or "Atg8p" and "hepatocellular carcinoma" or "HCC" or "liver cancer" or "hepatic tumor" or "liver tumor" or "hepatic cancer".

\section{Eligibility criteria}

Studies included in the meta-analysis had to meet the following inclusion criteria: (1) cohort or case-control design; (2) the patients were diagnosed with HCC and included clinicophathological or survival information; (3) LC3 expression was determined by immunohistochemistry (IHC). (4) The literature was published in English or Chinese and the full text was available.

The exclusion of studies were as follows: (1) case reports, reviews, letters, conference abstracts, simple commentaries, unpublished reports, (2) republished studies using the same dataset or patients, (3) the cohort or case-control design were unclear.

\section{Data extraction}

All data were assessed by two reviewers (Xiaoli Lou and Liyuan Yang) who extracted data that include the first author's name, publication year, sample size, country, type of cancer, detection method, patient characteristics, hazard ratio (HR) with $95 \%$ confidence interval (CI) for OS.

\section{Quality assessment}

Quality assessment was assessed using the Newcastle-Ottawa Scale (NOS). The NOS total scores ranged from 0 to 9 , and scores $1-3,4-6,7-9$ were defined as low-, moderate-, or high-quality studies, respectively. The ten articles included in this meta-analysis were considered to have a high quality.

\section{Statistical analysis}

The STATA software of version 14.2 (Stata Corporation, College Station, TX, USA) was performed in meta-analysis to assess the correlation between LC3 expression and clinicopathological features in HCC. Heterogeneity between the studies was tested using chi-squared test ( $Q$ test) and $I^{2}$ test. There was no significant heterogeneity when $p$ value $(Q$ test) $>0.1$ and $I^{2} \leq 50 \%$, the fixed-effects model was chosen; otherwise, a random-effects model was used. To explore the sources of heterogeneity, we conducted subgroup analyses based on sample size, NOS score, area and average age. Publication bias was assessed by Begg's test and funnel plot.

\section{Results}

A total of 1329 relevant studies were identified from the database, and 1281 studies were excluded based on screening of duplication, titles, abstracts, non-LC3 relation (Fig. 1). Full text of 48 articles was retrieved and assessed for eligibility. Among 48 studies, 38 were excluded because of ineligible and duplicated data about LC3 expression. Finally, ten publications were eligible for this meta-analysis (Wu et al. 2014a, b, 2016; Lee et al. 2013; Xi et al. 2013; Yu et al. 2018; Li et al. 2018; Zhao et al. 2016; Lu 2012; Song et al. 2014), all of which were cohort studies.

The characteristics of the included articles are summarized in Table 1. Four of the ten studies were written in English and six were in Chinese. These studies were published from 2012 to 2018 and a total of 949 patients with HCC were comprised. The number of patients ranged from 48 to $190 ; 6$ studies include $\leq 100$ patients, and 4 studies include $>100$ patients. Among the ten studies, nine were from China, one 
Fig. 1 Flow chart of study selection
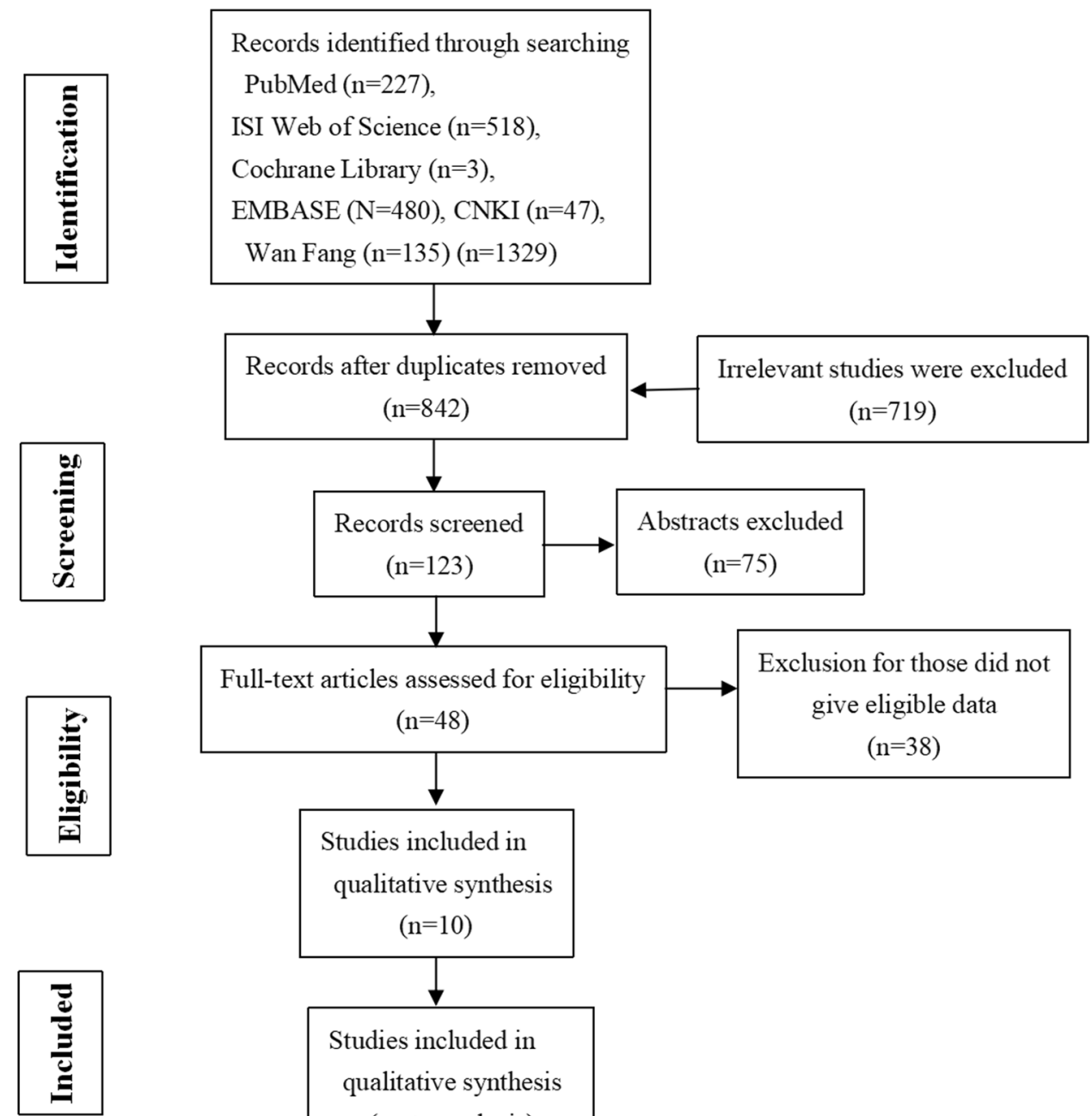

Records identified through searching

$(n=227)$

ISI Web of Science $(\mathrm{n}=518)$

Cochrane Library $(n=3)$,

EMBASE $(\mathrm{N}=480), \mathrm{CNKI}(\mathrm{n}=47)$

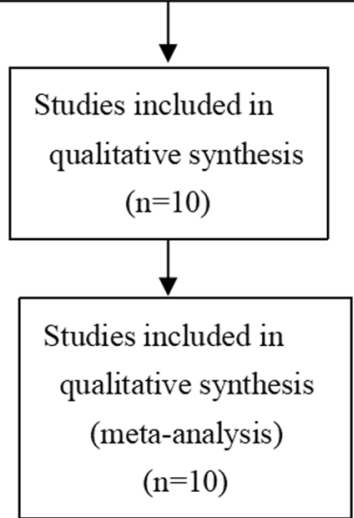

from Korea. LC3 expression were investigated through IHC staining. The quality of each included article was evaluated by NOS. These scores of studies were all $\geq 6$, indicating the high quality of included studies (Table 2).

\section{Meta-analysis of clinicopathological characteristics}

In this study, we assessed the correlation between LC3 expression and the clinicopathological features of HCC. As shown in Fig. 2 and Table 3, positive LC3 expression was positively associated with tumor size (OR 1.28, 95\% CI $[1.00,1.65], p=0.050$, fixed effect). However, the expression of LC3 was not associated with gender (OR 1.13, 95\% CI $[0.82,1.56], p=0.452$, fixed effect), age (OR 1.01, 95\% CI $[0.80,1.28], p=0.920$, fixed effect), number of tumors (OR 0.97, 95\% CI [0.67, 1.39], $p=0.838$, fixed effect), TNM stage (OR 0.90, 95\% CI [0.70, 1.16], $p=0.424$, fixed effect), alpha fetoprotein (AFP) (OR 1.22, 95\% CI [0.85, 1.73], $p=0.276$, fixed effect), vascular invasion (OR 1.17, 95\%
CI [0.84, 1.64], $p=0.357$, fixed effect), liver cirrhosis (OR $0.92,95 \%$ CI $[0.68,1.26], p=0.618$, fixed effect), HBsAg (OR 1.11, 95\% CI [0.83, 1.48], $p=0.483$, fixed effect) and histological grade (OR 0.95, 95\% CI [0.70, 1.28], $p=0.718$, random effect).

\section{Subgroup analysis}

As shown in Table 4, to explore the potential sources of heterogeneity, the subgroup analysis for histological grade and tumor size was performed using the sample size, NOS score, area and mean age. When the classifications of subgroups were based on sample size, LC3 expression was related to tumor size $(n>100$ : OR $1.39,95 \%$ CI [1.02, 1.88 ], $p=0.034$, random effect) in the large sample size but not in the subgroup of small sample size. Heterogeneity of histological grade were in the small sample size subgroup ( $\left.n \leq 100, I^{2}=66.9\right)$, and tumor size were in the large sample size subgroup $\left(n>100, I^{2}=66.1\right)$. However, 


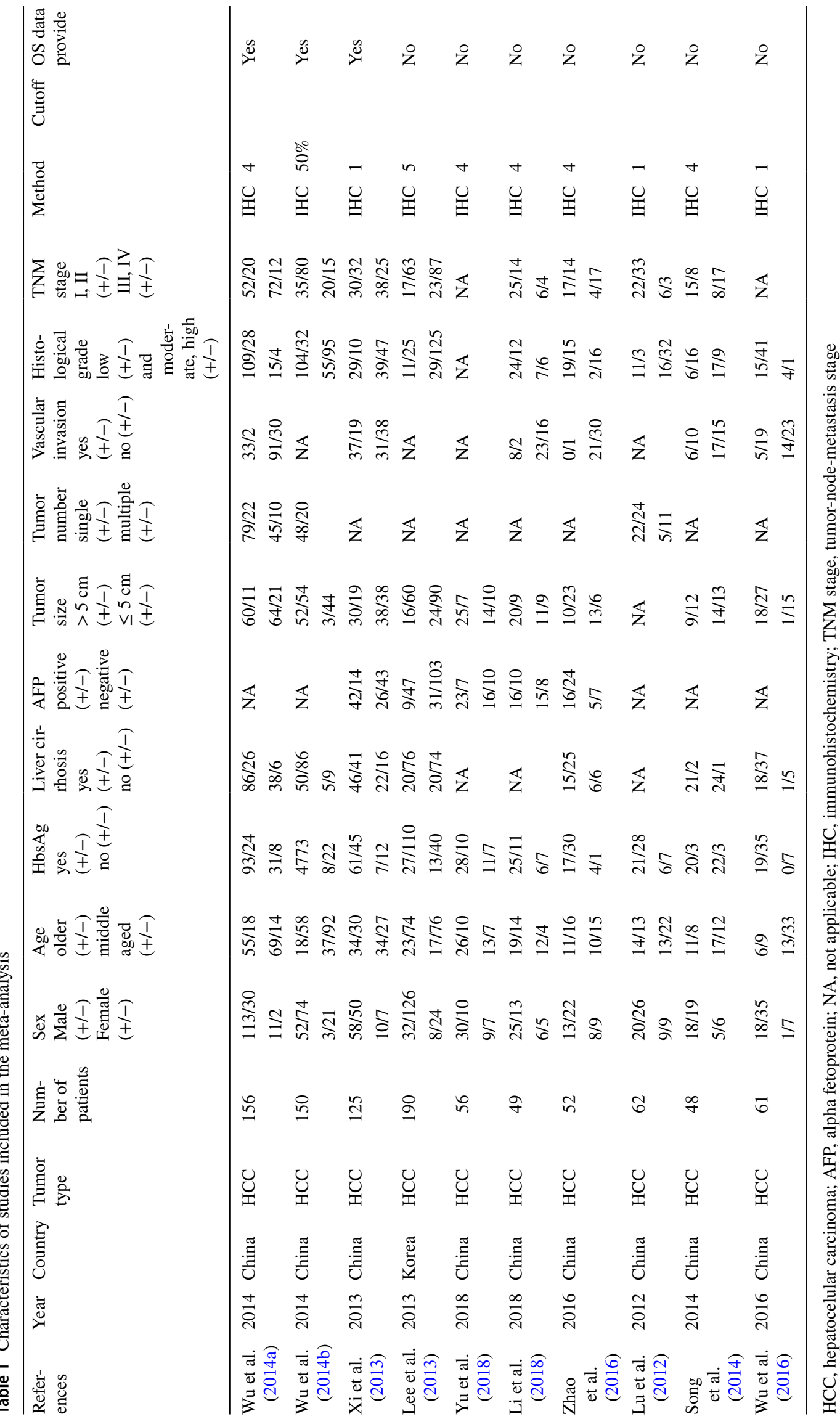


Table 2 Newcastle-Ottawa scale for quality assessment

\begin{tabular}{|c|c|c|c|c|c|c|c|c|c|}
\hline \multirow[t]{2}{*}{ Study } & \multicolumn{4}{|l|}{ Selection } & \multirow{2}{*}{$\begin{array}{l}\text { Comparability } \\
\text { Control for factor }\end{array}$} & \multicolumn{3}{|l|}{ Outcome } & \multirow[t]{2}{*}{ Total score } \\
\hline & $\begin{array}{l}\text { Exposed } \\
\text { cohort }\end{array}$ & $\begin{array}{l}\text { Non- } \\
\text { exposed } \\
\text { cohort }\end{array}$ & $\begin{array}{l}\text { Ascertain- } \\
\text { ment of } \\
\text { exposure }\end{array}$ & $\begin{array}{l}\text { Outcome } \\
\text { of interest }\end{array}$ & & $\begin{array}{l}\text { Assessment } \\
\text { of outcome }\end{array}$ & $\begin{array}{l}\text { Follow-up } \\
\text { long enough }\end{array}$ & $\begin{array}{l}\text { Adequacy } \\
\text { of follow-up }\end{array}$ & \\
\hline Dong-Hao Wu & $*$ & $*$ & $*$ & $*$ & $* *$ & $*$ & $*$ & $*$ & 9 \\
\hline Wen-Yong Wu & $*$ & $*$ & $*$ & $*$ & $* *$ & $*$ & $*$ & & 8 \\
\hline Shao-Yan Xi & $*$ & $*$ & $*$ & $*$ & $* *$ & $*$ & $*$ & $*$ & 9 \\
\hline Yoo Jin Lee & $*$ & $*$ & $*$ & $*$ & $* *$ & $*$ & $*$ & $*$ & 9 \\
\hline Yu Hai & $*$ & $*$ & $*$ & $*$ & $* *$ & $*$ & & & 7 \\
\hline Li Fang & $*$ & $*$ & $*$ & $*$ & $* *$ & $*$ & & & 7 \\
\hline Ya-Tong Zhao & $*$ & $*$ & $*$ & $*$ & $*$ & $*$ & $*$ & $*$ & 8 \\
\hline Yu-Dong Lu & $*$ & $*$ & $*$ & $*$ & $* *$ & $*$ & & & 7 \\
\hline Meng-Qi Song & $*$ & $*$ & $*$ & $*$ & $*$ & $*$ & & & 6 \\
\hline Wen-Yong Wu & * & $*$ & $*$ & * & $*$ & $*$ & * & $*$ & 8 \\
\hline
\end{tabular}

A study can be awarded one star for each numbered item within the selection and outcome categories. A maximum of two stars can be given for comparability. $* 1$ points of score are added in quality assessment. **2 points of score are added in quality assessment. https://www.ohri.ca/progr ams/clinical_epidemiology/oxford.asp

there was no heterogeneity in the tumor size with the small simple size and histological grade with the large simple size. The subgroup was classified by NOS score, LC3 expression was correlated with tumor size $(n>7$ : OR $1.33,95 \%$ CI $[1.00,1.76], p=0.051$, random effect). Heterogeneity was in both subgroups of histological grade $\left(n>7, I^{2}=52.9 ; n \leq 7, I^{2}=63.3\right)$. There was no heterogeneity in subgroup of tumor size $\left(n \leq 7, I^{2}=0.0\right)$. On the basis of the area, the LC3 expression was connected with tumor size (midland: OR 1.73, 95\% CI [1.04, 2.88], $p=0.035$, random effect) and histological grade (north: OR $0.94,95 \%$ CI $[0.51,1.75], p=0.035$, random effect; midland: OR $1.51,95 \%$ CI $[0.80,2.86], p=0.033$, random effect). There was no heterogeneity in southern subgroup of tumor size and histological grade. Subgroup analysis based on mean age showed that expression of LC3 was associated with tumor size (age $\geq 55$ : OR $1.81,95 \%$ CI $[1.22,2.70], p=0.003$, random effect). Heterogeneity was observed in tumor size subgroup (age $\geq 55, I^{2}=61.0$ ) and histological grade subgroup (age $<55, I^{2}=66$ ). Results of subgroups analysis revealed that the sample size, NOS score, area and mean age score most likely caused heterogeneity in tumor size and histological grade.

\section{Correlation of LC3 expression with overall survival (OS)}

Based on meta-analysis, we evaluated the prognostic value of LC3 that high LC3 expression was correlation with the overall survival (HR 1.809, 95\% CI [1.164, 2.812], $p=0.008$, fixed effect) (Fig. 3).

\section{Publication bias}

As shown in Fig. 4, the Begg's test was used to evaluate potential publication bias. No publication bias was confirmed to exist in gender $(p=0.074)$, age $(p=0.474)$, liver cirrhosis $(p=0.548), \mathrm{HBsAg}(p=0.592)$, number of tumors $(p=0.296)$, size of tumors $(p=0.466)$, TNM stage $(p=0.266)$, AFP $(p=0.806)$, OS $(p=0.602)$, vascular invasion $(p=0.133)$, histological grade $(p=0.348)$. We conduct a sensitivity analysis by excluding one study in turn. Interestingly, heterogeneity of histological grade declined from $I^{2}=50.3$ to $I^{2}=37.6$ after removing the article from Zhao et al. (2016).

\section{Discussion}

Autophagy plays a crucial role in immune responses, cellular metabolism, apoptosis, and cell death (Choi et al. 2013). It has been reported that autophagy remains multiple roles in tumor, it may promote tumor progression and also can inhibit tumor transformation (Morselli et al. 2009). The attention of the relationship between autophagy and cancer have been increased. Recent studies have focused on evaluating the clinicopathological and prognostic values of autophagy-related markers in 
Fig. 2 Forest plot of studies assessing the relationship between LC3 expression and a gender, $\mathbf{b}$ age, $\mathbf{c}$ liver cirrhosis, d HbsAg, e tumor number, $\mathbf{f}$ AFP, $\mathbf{g}$ tumor size, $\mathbf{h}$ TNM stage, i vascular invasion, $\mathbf{j}$ histological grade. $C I$ confidence interval; $O R$ odds ratio
A

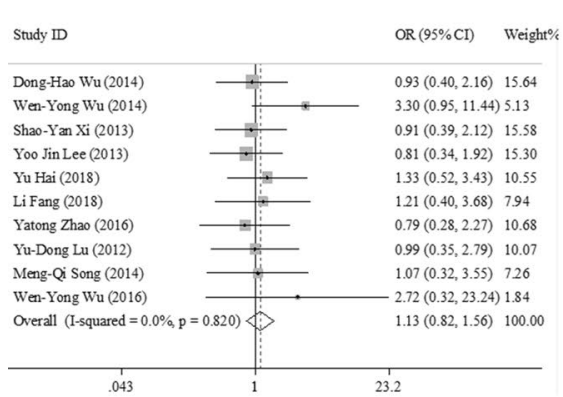

C

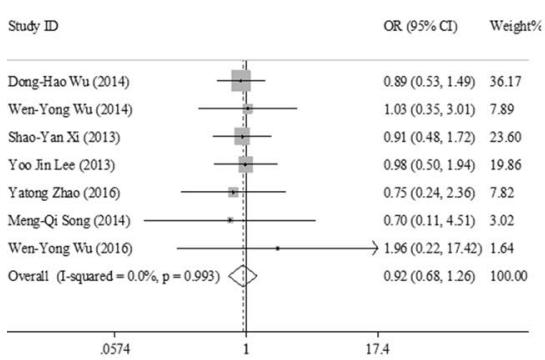

$\mathbf{E}$

Study ID

OR (95\% CI) Weighe\%

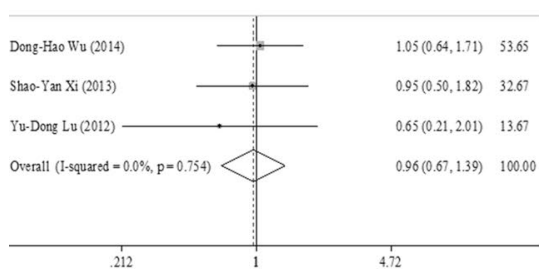

G

Sudy D

Dong-Hao Wu (2014)

Wen-Y ong Wu (2014)

Shao-Yan Xi (2013)

Yoo In Lee (2013)

YuHai (2018)

Li Fang (2018)

Yatong Zhao (2016)

Meng-Qi Song (2014)

Wen-Yong Wu (2016)

$\longrightarrow 6.40(0.79,51.89) 1.03$

\begin{tabular}{l|l}
1.28 & $(1.00,1.65) 100.00$ \\
\hline
\end{tabular}

I

Study ID

OR $(95 \% \mathrm{CI})$

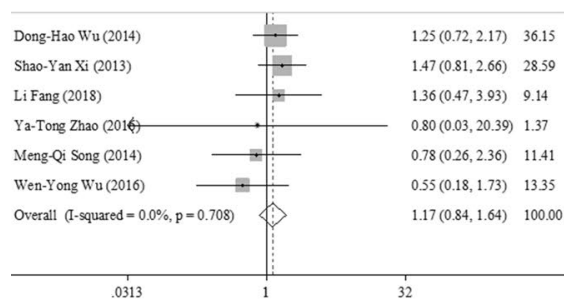

OR $(95 \% \mathrm{CI}) \quad$ Weight $\%$

$1.12(0.70,1.80) 29.79$

$7.19(2.13,24.27) 2.85$

$1.22(0.67,2.23) 17.71$

$1.00(0.50,2.01) 14.56$

$1.34(0.58,3.11) 8.66$

$1.25(0.49,3.18) 7.32$

$0.44(0.16,1.20) \quad 10.50$

.
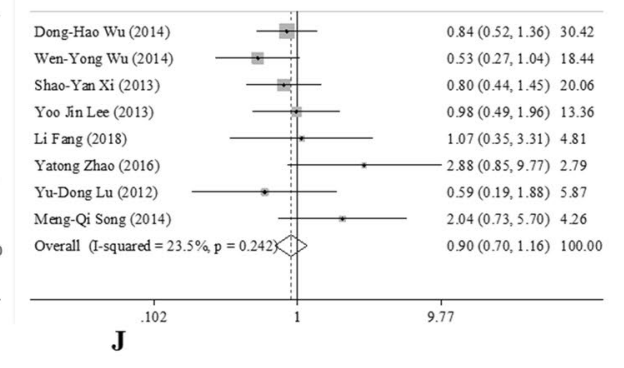

$\mathbf{J}$

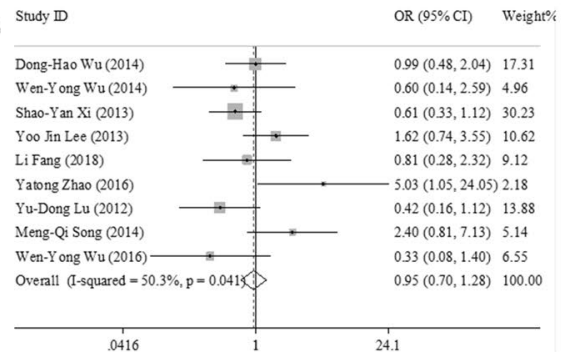

Sudy D

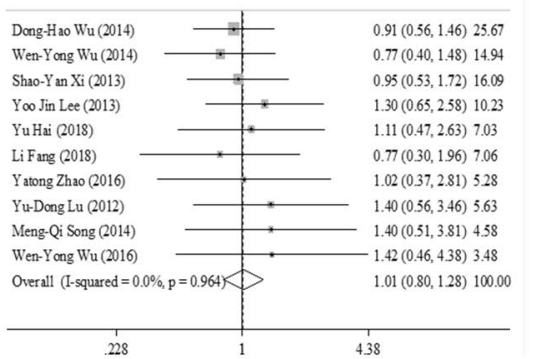

D

Study ID

OR $(95 \%$ CD) Weighe\%

Dong-Hao Wu (2014) $1.00(0.58,1.72) 2923$

Wen-Yong Wu (2014)

Shao-Yan Xi (2013)

Yoo In Lee (2013)

Yu Hai (2018)

Li Fang (2018)

Yatong Zhao (2016)

Meng-Qi Song (2014)

Meng-Q1 Song (2014)

$1.21(0.49,2.95) 9.93$

$1.50(0.50,4.49) 6.09$

$0.45(0.11,1.88) 5.81$

$0.93(0.31,2.77) 7.46$

$5.37(0.29,98.43) 0.75$

.0102

98.4

F

Study ID $\quad \mathrm{OR}(95 \% \mathrm{Cl}) \quad$ Weight $\%$

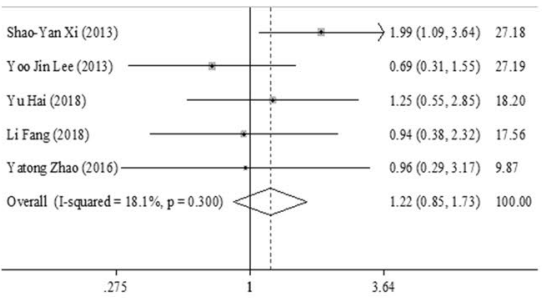

H

Study ID

OR $(95 \% \mathrm{Cl}) \quad$ Weight\%

1.36) 30.42 1.45) 20.06 $1.07(0.35,3.31) 4.81$ $88(0.85,9.77) 2.79$ $59(0.19,1.88) 5.87$ $2.04(0.73,5.70) 4.26$
$0.90(0.70,1.16) 100.00$

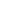

.


Table 3 LC3 clinicopathological features for HCC

\begin{tabular}{|c|c|c|c|c|c|c|c|}
\hline \multirow{2}{*}{ Clinicopathological features } & \multirow{2}{*}{$\begin{array}{l}\text { Number of } \\
\text { studies }\end{array}$} & \multirow{2}{*}{$\begin{array}{l}\text { Number of } \\
\text { patients }\end{array}$} & \multirow{2}{*}{ Pooled OR $(95 \% \mathrm{CI})$} & \multirow{2}{*}{$\begin{array}{l}\text { Meta-regression } \\
p \text { value }\end{array}$} & \multicolumn{2}{|c|}{ Heterogeneity } & \multirow[t]{2}{*}{ Model used } \\
\hline & & & & & $p$ value & $I^{2}(\%)$ & \\
\hline Gender & 10 & 949 & $1.13(0.82,1.56)$ & 0.452 & 0.820 & 0.0 & Fixed \\
\hline Age & 10 & 949 & $1.01(0.80,1.28)$ & 0.920 & 0.964 & 0.0 & Fixed \\
\hline HbsAg & 10 & 949 & $1.11(0.83,1.48)$ & 0.483 & 0.839 & 0.0 & Fixed \\
\hline AFP & 5 & 472 & $1.22(0.85,1.73)$ & 0.276 & 0.300 & 18.1 & Fixed \\
\hline Liver cirrhosis & 7 & 782 & $0.92(0.68,1.26)$ & 0.618 & 0.993 & 0.0 & Fixed \\
\hline Tumor size & 9 & 887 & $1.28(1.00,1.65)$ & 0.050 & 0.044 & 49.7 & Fixed \\
\hline Tumor number & 3 & 343 & $0.97(0.67,1.39)$ & 0.838 & 0.754 & 0.0 & Fixed \\
\hline Vascular invasion & 6 & 491 & $1.17(0.84,1.64)$ & 0.357 & 0.708 & 0.0 & Fixed \\
\hline Histological grade & 9 & 893 & $0.95(0.70,1.28)$ & 0.718 & 0.004 & 50.3 & Random \\
\hline TNM stage & 8 & 783 & $0.90(0.70,1.16)$ & 0.424 & 0.242 & 23.5 & Fixed \\
\hline
\end{tabular}

,Fixed fixed-effects model, Random random-effects model

Table 4 Subgroup analysis of histological grade and tumor size by sample size, NOS score, area and average age

\begin{tabular}{|c|c|c|c|c|c|c|c|}
\hline Subgroups & $\begin{array}{l}\text { Number of } \\
\text { studies }\end{array}$ & $\begin{array}{l}\text { Number of } \\
\text { patients }\end{array}$ & Pooled OR $(95 \% \mathrm{CI})$ & $p$ value & PHet & $I^{2}(\%)$ & Model used \\
\hline \multicolumn{8}{|l|}{ Tumor size } \\
\hline \multicolumn{8}{|l|}{ Sample } \\
\hline$n>100$ & 4 & 621 & $1.39(1.02,1.88)$ & 0.034 & 0.031 & 66.1 & Random \\
\hline$n \leq 100$ & 5 & 218 & $1.09(0.70,1.69)$ & 0.697 & 0.166 & 38.2 & Fixed \\
\hline \multicolumn{8}{|l|}{ NOS score } \\
\hline$>7$ & 6 & 734 & $1.33(1.00,1.76)$ & 0.051 & 0.009 & 67.6 & Random \\
\hline$\leq 7$ & 3 & 105 & $1.15(0.68,1.95)$ & 0.610 & 0.753 & 0.0 & Fixed \\
\hline \multicolumn{8}{|l|}{ Area } \\
\hline North & 2 & 246 & $1.13(0.66,1.92)$ & 0.662 & 0.600 & 0.0 & Fixed \\
\hline Midland & 4 & 311 & $1.73(1.04,2.88)$ & 0.035 & 0.001 & 81.2 & Random \\
\hline South & 3 & 330 & $1.17(0.83,1.66)$ & 0.364 & 0.964 & 0.0 & Fixed \\
\hline \multicolumn{8}{|l|}{ Average age } \\
\hline$\geq 55$ & 5 & 506 & $1.81(1.22,2.70)$ & 0.003 & 0.036 & 61.0 & Random \\
\hline$<55$ & 4 & 381 & $1.01(0.73,1.40)$ & 0.967 & 0.340 & 10.6 & Fixed \\
\hline \multicolumn{8}{|c|}{ Histological grade } \\
\hline \multicolumn{8}{|l|}{ Sample } \\
\hline$n>100$ & 4 & 621 & $0.88(0.60,1.31)$ & 0.536 & 0.251 & 26.8 & Fixed \\
\hline$n \leq 100$ & 5 & 272 & $1.05(0.64,1.71)$ & 0.843 & 0.017 & 66.9 & Random \\
\hline \multicolumn{8}{|l|}{ NOS score } \\
\hline$>7$ & 6 & 734 & $0.96(0.67,1.37)$ & 0.822 & 0.059 & 52.9 & Random \\
\hline$\leq 7$ & 3 & 159 & $0.91(0.51,1.62)$ & 0.746 & 0.065 & 63.3 & Random \\
\hline \multicolumn{8}{|l|}{ Area } \\
\hline North & 2 & 252 & $0.94(0.51,1.75)$ & 0.854 & 0.035 & 77.5 & Random \\
\hline Midland & 4 & 311 & $1.51(0.80,2.86)$ & 0.206 & 0.033 & 65.7 & Random \\
\hline South & 3 & 330 & $0.76(0.49,1.16)$ & 0.205 & 0.596 & 0.0 & Fixed \\
\hline \multicolumn{8}{|l|}{ Average age } \\
\hline$\geq 55$ & 4 & 450 & $0.95(0.55,1.64)$ & 0.859 & 0.230 & 30.3 & Fixed \\
\hline$<55$ & 5 & 443 & $0.94(0.65,1.36)$ & 0.751 & 0.019 & 66.0 & Random \\
\hline
\end{tabular}

NOS Newcastle-Ottawa Scale 


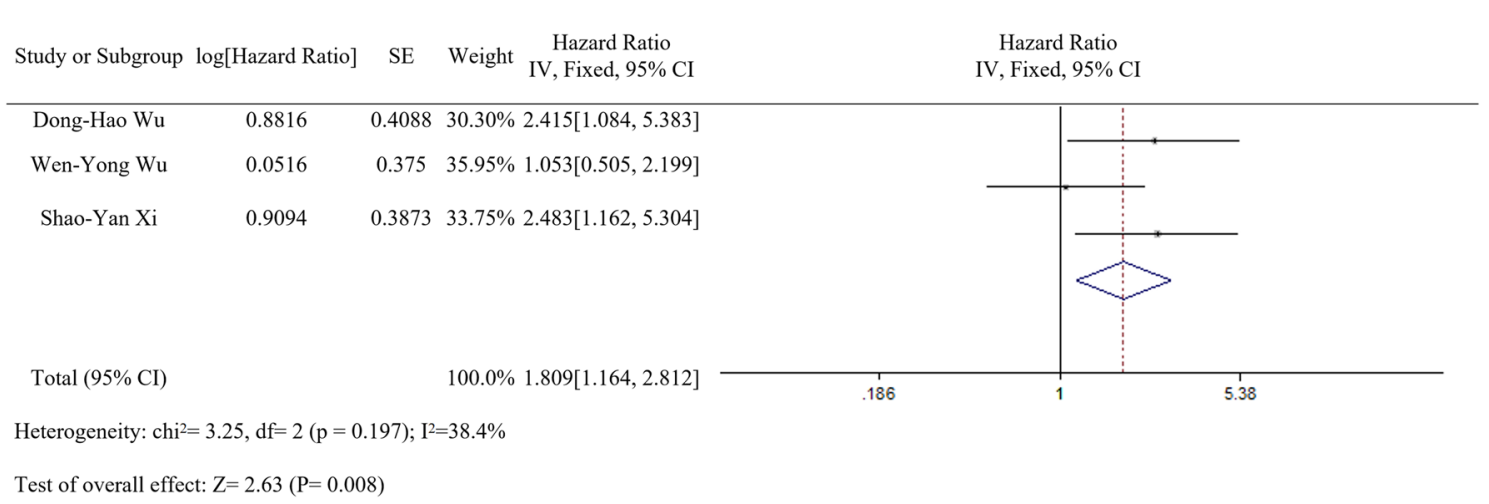

Fig. 3 Forest plot of studies assessing the relationship between LC3 expression andOS in HCC patients

cancer revealed their clinical significances. LC3 is identified autophagy-related gene that plays a crucial role in the process of tumorgenesis (Galais et al. 2019). Currently, the role of LC3 in HCC is controversial reported. Therefore, we performed this meta-analysis to evaluating clinicopathological and prognostic values of LC3 in HCC.

In this meta-analysis, 949 patients from 10 studies were performed. Positive LC3 expression is related to tumor size but not to gender, age, number of tumors, HBsAg, liver cirrhosis, TNM stage, AFP, vascular invasion and histological grade. The relationship between LC3 expression and tumor size was explored, using 887 patients from 9 articles. Eight of the nine studies showed that LC3 expression was not associated with tumor size, but the pooled results showed a positive correlation between LC3 expression and tumor size. Tumor growth can cause the ischemic and hypoxic environment, LC3 may alleviate this state and enhance the environmental viability of tumor. The increase in tumor diameter induces a sustained increase in LC3 levels in tumor cells, which in turn induces autophagic recycle, provides nutrients for the tumor cells, and promotes tumor proliferation. Thus, this result indicates that LC3 may plays an important role in the occurrence and development of HCC. Moreover, our data indicated that high LC3 expression level is related to the overall survival in HCC, which also confirmed by Wu et al. (2014a) and Lee et al. (2013) studies. Therefore, the change of LC3 level may be related to the occurrence, evolution and poor prognosis of tumor, suggesting that the expression of LC3 is closely related to the occurrence and development of HCC.

This study has been the first comprehensive and systematic meta-analysis exploring the relationship between LC3 and its clinicopathological fators in HCC. Recent reports show that the combination of other related genes can improve clinical predictive value. Zhao et al. (2017) have found that beclin 1 and LC 3 can be potential prognostic markers in retrospective studies of ovarian cancer. $\mathrm{Wu}$ et al. (2018) have demonstrated that ULK1 combined with LC3B would improve prognosis assessment of the HCC patients and so on. Thus, LC3 can also be combined with other related genes for clinical diagnosis.

Significant heterogeneity with the correlation of LC3 expression and histological grade or tumor size was determined in this analysis. Sensitivity and subgroup analysis were performed to explore the sources of the heterogeneities. Subgroup analysis based on NOS showed that LC3 expression was uncorrelated with histological grade of HCC patients. The heterogeneity existed in subgroup of tumor size $(n \leq 7)$. The subgroups were classified by sample size, LC3 expression was related to tumor size $(n>100)$ but not to histological grade. On the basis of the area, heterogeneity was both in midland subgroup of tumor size and histological grade. Subgroup analysis based on mean age, heterogeneity was observed in tumor size subgroup (age $\geq 55$ ) and histological grade subgroup (age $<55$ ). This suggests that the reasons leading to the heterogeneity have many different characteristics. First, the detection methods and publication years are different. Second, IHC, a semi-quantitative assessment method, which is affected by many factors such as the concentration and incubation time of the antibody and diaminobezidin, the quality of the antibody, and so on, some of which are based on individual manners. This may be a potential factor for the occurrence of heterogeneity. Third, diverse sample sources exist in each study. Finally, lack of eligible articles for this meta-analysis. Therefore, more highquality and large-scale studies on the relationship of LC3 and HCC should be performed in the future.

\section{Limitations of the study}

Our meta-analysis has some limitations including: (1) the number of included articles was less, more high-quality studies need to be included; (2) included studies have different 
Fig. 4 Funnel plot of studies assessing publication bias of the relationship betweenLC3 expression and a gender, $\mathbf{b}$ age, c liver cirrhosis, d HbsAg, e tumor number, fAFP, $\mathbf{g}$ tumor size, $\mathbf{h}$ TNM stage, $\mathbf{i}$ vascular invasion, $\mathbf{j}$ histological grade
A
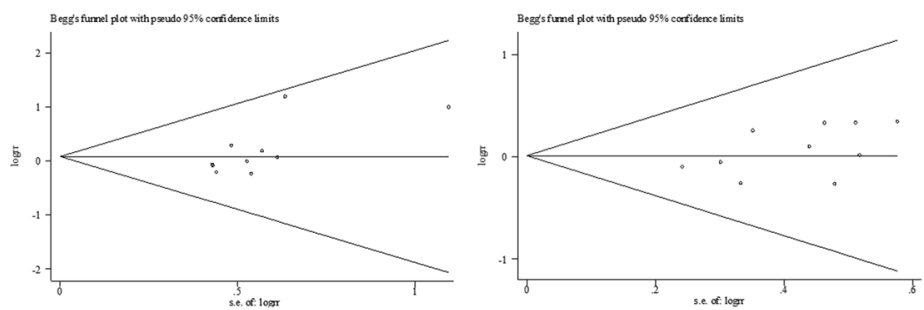

C

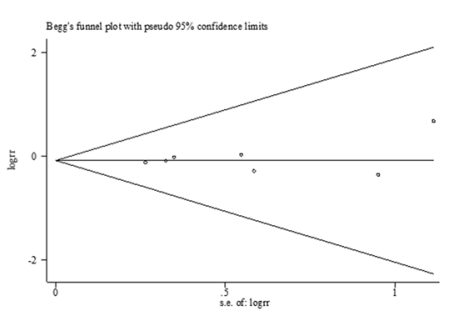

E

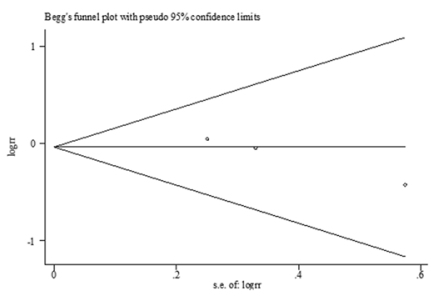

G
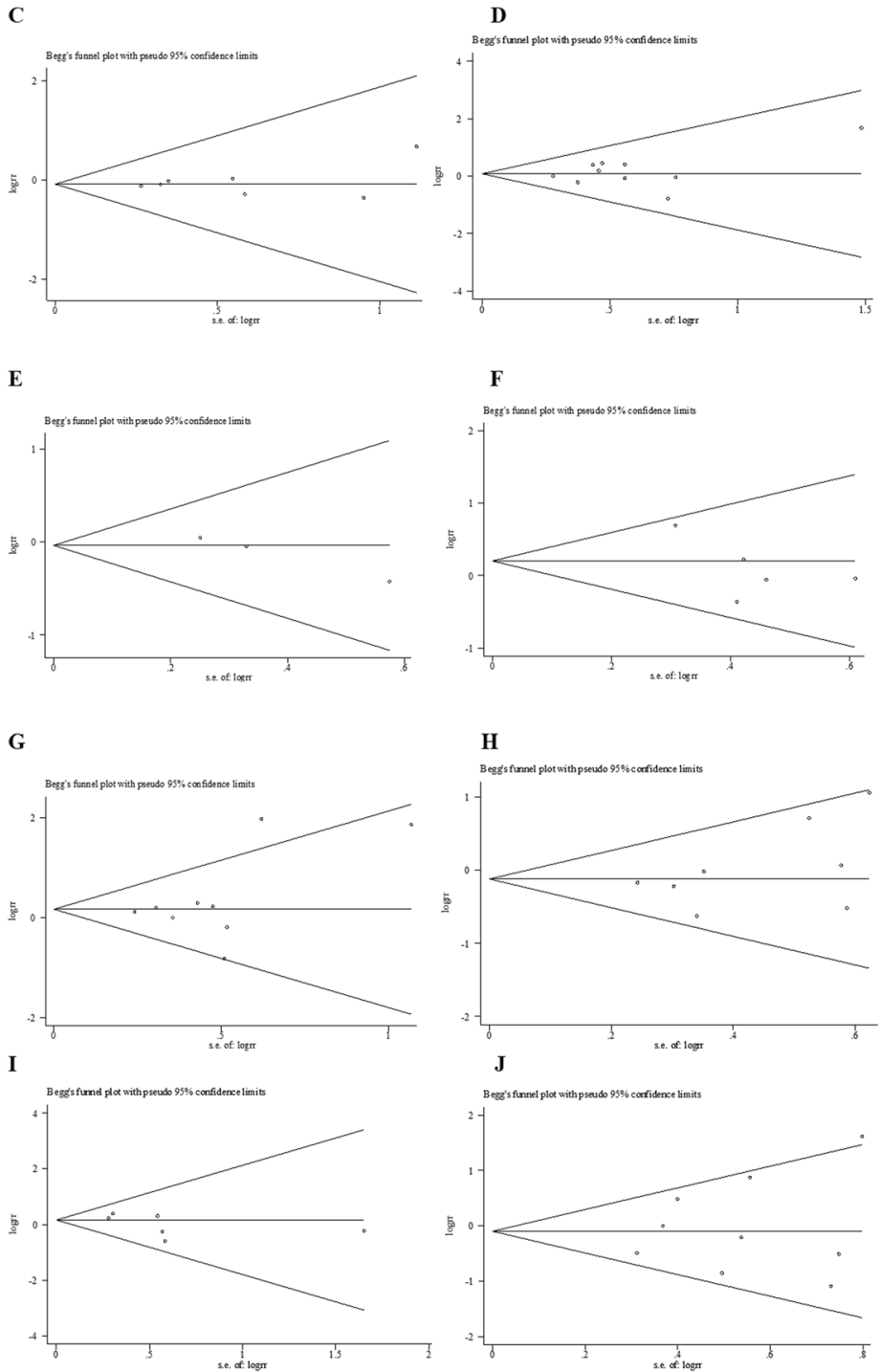

F

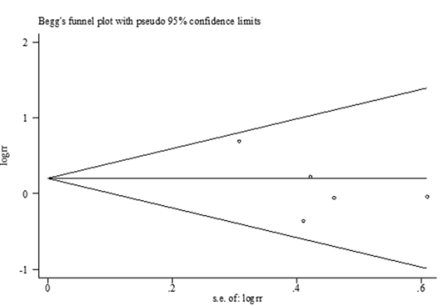

H

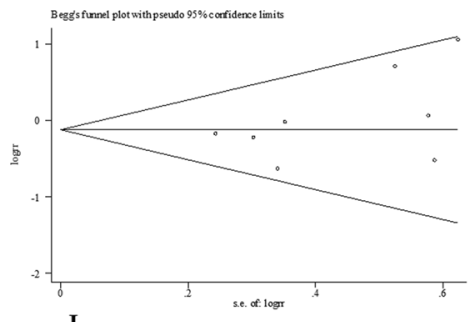

J

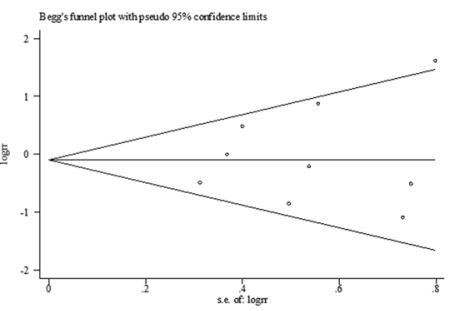

$\mathbf{K}$

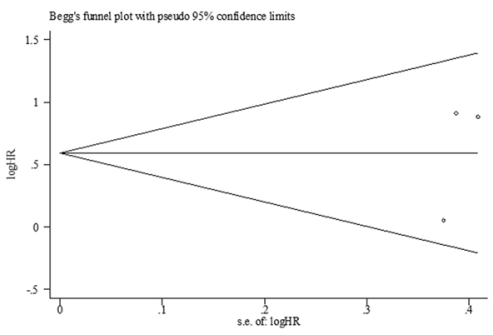


cut-off values for IHC scores, this may lead to a potential heterogeneity. The exploration of heterogeneity may have been inadequate, due to the limited variables collected from included studies; (3) of the included studies, nine were from China and one was from Korea, studies from other regions were not available; (4) some individual research data could not be obtained, and the analysis of the prognostic role of LC3 included only three studies, the research volume was relatively small.

\section{Conclusions}

In summary, our meta-analysis indicated that positive LC3 is only positively correlated with tumor size in clinicopathological features of HCC. LC3 can be used a prognostic marker in $\mathrm{HCC}$ and combined with other related genes may improve clinical value. However, these results need to be confirmed by more large sample size and high-quality articles.

Acknowledgements This work was supported by National Natural Science Foundation of China (NSFC; no. 81702729), Shanghai Songjiang District Science and Technology Research Project (no. 18sjkjggbb) and Shanghai Municipal Health Bureau Project (no. 201940025).

Author contributions All authors contributed to this work. Study design: Y-QH. Writing, constitute figures and tables and data analysis: Y-CM. Screening of abstracts and articles: X-LL and L-YY. Critically reviewing manuscript: DL and Y-QH.

\section{Compliance with ethical standards}

Conflict of interest The authors declare that they have no conflict of interest.

Open Access This article is licensed under a Creative Commons Attribution 4.0 International License, which permits use, sharing, adaptation, distribution and reproduction in any medium or format, as long as you give appropriate credit to the original author(s) and the source, provide a link to the Creative Commons licence, and indicate if changes were made. The images or other third party material in this article are included in the article's Creative Commons licence, unless indicated otherwise in a credit line to the material. If material is not included in the article's Creative Commons licence and your intended use is not permitted by statutory regulation or exceeds the permitted use, you will need to obtain permission directly from the copyright holder. To view a copy of this licence, visit http://creativecommons.org/licenses/by/4.0/.

\section{References}

Bao L, Chandra PK, Moroz K et al (2014) Impaired autophagy response in human hepatocellular carcinoma. Exp Mol Pathol 96:149-154

Boyvat F (2017) Interventional radiologic treatment of hepatocellular carcinoma. Exp Clin Transplant 15(suppl 2):25-30
Chih WL, Chih CH, Lee PH et al (2017) The autophagy marker LC3 strongly predicts immediate mortality after surgical resection for hepatocellular carcinoma. Oncotarget 8:91902-91913

Choi AM, Ryter SW, Levine B (2013) Autophagy in human health and disease. N Engl J Med 368:651-662

Ding ZB, Shi YH, Zhou J et al (2008) Association of autophagy defect with a malignant phenotype and poor prognosis of hepatocellular carcinoma. Cancer Res 68:9167-9175

Galais M, Pradel B, Vergne I et al (2019) LAP (LC3-associated phagocytosis): phagocytosis or autophagy? Med Sci (Paris) 35:635-642

Ghavami S, Shojaei S, Yeganeh B et al (2014) Autophagy and apoptosis dysfunction in neurodegenerative disorders. Prog Neurobiol 112:24-49

Gingold JA, Zhu D, Lee DF et al (2018) Genomic profiling and metabolic homeostasis in primary liver cancers. Trends Mol Med 24:395-411

Grandhi MS, Kim AK, Ronnekleiv-Kelly SM et al (2016) Hepatocellular carcinoma: from diagnosis to treatment. Surg Oncol 25:74-85

Han Y, Xue XF, Shen HG et al (2014) Prognostic significance of Beclin-1 expression in colorectal cancer: a meta-analysis. Asian Pac J Cancer Prev 15:4583-4587

Hartke J, Johnson M, Ghabril M (2017) The diagnosis and treatment of hepatocellular carcinoma. Semin Diagn Pathol 34:153-159

Jiang X, Bao Y, Liu H et al (2017) VPS34 stimulation of p62 phosphorylation for cancer progression. Oncogene 36:6850-6862

Lee YJ, Jang BK (2015) The role of autophagy in hepatocellular carcinoma. Int J Mol Sci 16:26629-26643

Lee Y, Hah YJ, Kang YN et al (2013) The autophagy-related marker LC3 can predict prognosis in human hepatocellular carcinoma. PLoS ONE 8:e81540

Li F, Xu P, Gao TT et al (2018) Expression and significance of autophagy related protein Beclin1, LC3, and mTOR in hepatic carcinoma. Chin J Gerontol 8:3890-3892

Liang CJ, Ge H, Zhang KT et al (2018) Role of beclin1 expression in patients with hepatocellular carcinoma: a meta-analysis. OncoTargets Ther 4:2387-2397

Lu YD (2012) Expression and significance of autophagy related protein LC3. MA thesis. Taiyuan: Shanxi medical University, China

Lv C, Zhang Z, Zhao T et al (2019) The anti-tumour effect of Mel and its role in autophagy in human hepatocellular carcinoma cells. Am J Transl Res 11:931-941

Maruyama Y, Sou S, Kageyam S et al (2014) LC3B is indispensable for selective autophagy of p62 but not basal autophagy. Biochem Biophys Res Commun 446:309-315

Morselli E, Galluzzi L, Kepp O et al (2009) Anti- and pro-tumor functions of autophagy. Biochim Biophys Acta 1793:1524-1532

Segala G, David M, de Medina P et al (2017) Dendrogenin A drives LXR to trigger lethal autophagy in cancers. Nat Commun 8:1903

Shen Y, Liang LZ, Hong MH et al (2008) Expression and clinical significance of microtubule-associated protein 1 light chain 3 (LC3) and Beclin1 in epithelial ovarian cancer. Ai Zheng 27:595-599

Song MQ, Xue YK, Sun YX et al (2014) Expression and clinical significance of mammalian target of rapamycin and microtubulesassociated protein 1 light chain 3- $\beta$ in hepatocellular carcinama. Chin J Exp Surg 31:1108-1110

Wu DH, Jia CC, Chen J et al (2014a) Autophagic LC3B overexpression correlates with malignant progression and predicts a poor prognosis in hepatocellular carcinoma. Tumor Biol 35:12225-12233 
Wu WY, Kim H, Zhang CL et al (2014b) Clinical signification of autophagic protein LC3 levels and its correlation with XIAP expression in hepatocellular carcinoma. Med Oncol 31:108

Wu S, Sun C, Tian D et al (2015) Expression and clinical significances of Beclin1, LC3 and mTOR in colorectal cancer. Int J Clin Exp Pathol 8:3882-3891

Wu WY, Meng XL, Qi Y et al (2016) Clinical significance of LC3B and p62 expression in human hepatocellular carcinoma. J Clin Exp Pathol 32:366-369

Wu DH, Wang TT, Ruan DY et al (2018) Combination of ULK1 and LC3B improve prognosis assessment of hepatocellular carcinoma. Biomed Pharmacother 97:195-202

Xi SY, Lu JB, Chen JW et al (2013) The "stone-like" pattern of LC3A expression and its clinicopathologic significance in hepatocellular carcinoma. Biochem Biophys Res Commun 431:760-766

Yao LL, Zhou YD, Sui AH et al (2019) HBV-encoded miR-2 functions as an oncogene by downregulating TRIM35 but upregulating RAN in liver cancer cells. EBioMedicine 09:1-13
Yu H, Liu QH, Xiao PL et al (2018) Expression and clinical significance of Beclin1, LC3, and mTOR in hepatic carcinoma. Anhui Med Pharm J 22:246-249

Zhang S, Li J, Zhou G et al (2017) Aurora-A regulates autophagy through the Akt pathway in human prostate cancer. Cancer Biomark 19:27-34

Zhao YT, Pang ZG, Ma DM et al (2016) Expression of forkhead transcription factor group $\mathrm{O} 1$ and autophagy related protein microtubule associated protein 1 light chain 3-II in hepatocellular carcinama and significance. Chin J Exp Surg 33:492-494

Zhao Z, Xue J, Zhao X et al (2017) Prognostic role of autophagyrelated proteins in epithelial ovarian cancer: a meta-analysis of observational studies. Minerva Med 108:277-286

Publisher's Note Springer Nature remains neutral with regard to jurisdictional claims in published maps and institutional affiliations. 